\title{
MyoD, modularity, and myogenesis: conservation of regulators and redundancy in C. elegans
}

\author{
L. Ryan Baugh ${ }^{1,4}$ and Craig P. Hunter ${ }^{2,3}$ \\ ${ }^{1}$ Howard Hughes Medical Institute and Division of Biology, California Institute of Technology, Pasadena, California 91125 , \\ USA; ${ }^{2}$ Department of Molecular and Cellular Biology, Harvard University, Cambridge, Massachusetts 02138, USA
}

The positive autoregulatory circuits . . . though potentially very complex, seem designed to commit cells to the myogenic pathway.

—Weintraub 1993

In a now classic example of genetic redundancy, overexpression of the basic helix-loop-helix (bHLH) transcription factor MyoD or other members of the MyoD family of myogenic regulatory factors (MRFs) can transform numerous cell types into muscle, but homozygous knockout of either MyoD or other MRFs alone in mice results in fairly normal myogenesis (Weintraub et al. 1989; Braun et al. 1992; Mak et al. 1992; Rudnicki et al. 1992). That no muscle is formed in double mutants (Rudnicki et al. 1993) where the function of two or three MRFs is eliminated (Kassar-Duchossoy et al. 2004) demonstrates that MyoD and other MRFs have overlapping, or redundant, function (Rudnicki et al. 1993; Weintraub 1993). Genes with completely overlapping function cannot be maintained across large evolutionary time, and MyoD and Myf5 knockout mutants do in fact have different, albeit relatively subtle, phenotypes (Kablar et al. 1997; Ordahl and Williams 1998). Weintraub (1993) first suggested that the overlapping function and reciprocal regulation of MyoD and Myf5 result in a genetic circuit with switch-like behavior to irreversibly commit cells to the myogenic pathway. Other transcriptional regulators have also been implicated in vertebrate myogenesis, in particular, the additional MRFs and members of the MADS/MEF2 family, and with their characterization the model of a highly cooperative network controlling muscle development has been further substantiated (Molkentin and Olson 1996; Yun and Wold 1996). Recent work in the nematode Caenorhabditis elegans (Fukushige et al. 2006) reinforces parallels between vertebrate and invertebrate myogenesis, demonstrating conservation of regulators and redundancy, while resolving a long-standing paradox between vertebrate and invertebrate myogenesis.

Correspondence.

${ }^{3}$ E-MAIL hunter@mcb.harvard.edu; FAX (617) 496-0132.

${ }^{4}$ E-MAIL 1rb@caltech.edu; FAX (626) 568-8012.

Article is online at http://www.genesdev.org/cgi/doi/10.1101/gad.1507606.
The essential role of vertebrate bHLH MRFs in myogenesis led to surprise when it was found in both the nematode C. elegans and the fruitfly Drosophila melanogaster that the single MyoD homolog (hlh-1 and nautilus, respectively) is not required for muscle specification (Paterson et al. 1991; Chen et al. 1992). A study by Fukushige et al. (2006) in this issue of Genes \& Development conclusively demonstrates that hlh-1 does control muscle specification in C. elegans, but as shown previously, its function is masked by genetic redundancy (Baugh et al. 2005b). Fukushige et al. (2006) corroborate and extend previous results (Baugh et al. 2005a,b) demonstrating that hlh-1 (CeMyoD), unc-120 (MADS-box/ $\mathrm{SRF})$, and hnd-1 (HAND/bHLH) redundantly control muscle specification in C. elegans. Together these three genes comprise a functionally robust "muscle module" with implications for the evolution of contractile cell types as well as the dynamics and robustness of muscle cell fate specification. In particular, the closest vertebrate homologs of unc-120 and hnd-1 (SRF and HAND) play prominent roles regulating smooth and cardiac muscle development but not skeletal, which is homologous to the body wall muscle of $C$. elegans. The fact that all three factors are found to coordinately regulate body wall muscle development led Fukushige et al. (2006) to suggest a common evolutionary origin for all muscle followed by divergence, rather than multiple, independent origins of muscle (see Fig. 1). Furthermore, evolution has favored overlapping function and reciprocal regulation among multiple myogenic regulators, along with the regulators themselves. We thus extend their suggestion and propose that such a redundant, modular network controlling myogenesis allows for dynamic compensation of function, rendering muscle development robust and perhaps even enabling evolution of different types of muscle.

\section{C. elegans MyoD homolog hlh-1 is not essential to muscle specification}

When CeMyoD (hlh-1) was first cloned from C. elegans, analysis of its sequence conservation and expression in early myoblasts was consistent with it being a critical 


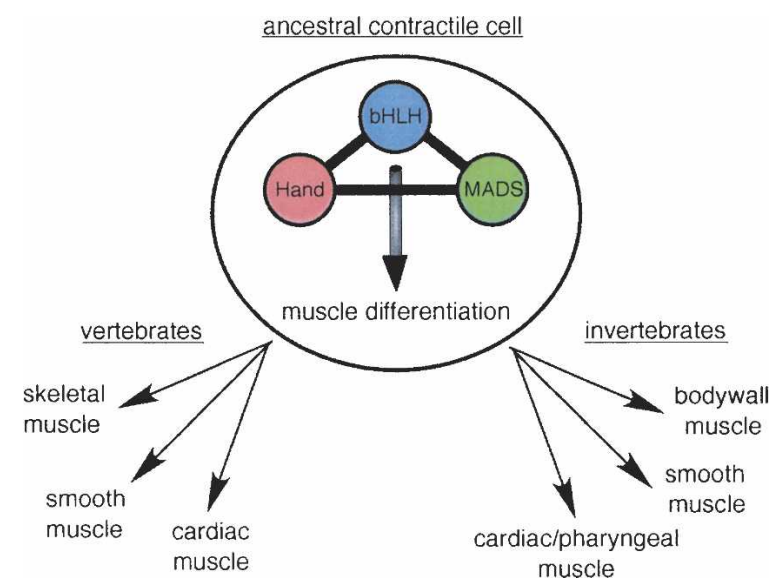

Figure 1. A unified theory of animal muscle development proposed by Fukushige et al. (2006). Genetic analysis of muscle development in C. elegans suggests that members of each of the transcription factor families that control myogenesis in modern animals also controlled muscle differentiation in an ancestral contractile cell and that this common ancestor gave rise to all of the muscle types found today in vertebrates and invertebrates.

early regulator of muscle specification (Krause et al. 1990). But while null mutations in hlh-1 were found to affect viability by disrupting post-embryonic muscle function (Chen et al. 1994), early muscle development appeared grossly normal, indicating that $h l h-1$ is not essential for embryonic muscle specification (Chen et al. 1994). The fact that near normal numbers of muscle cells are made and differentiate in hlh-1 mutants suggested that $h l h-1$ functions redundantly with other unidentified factors to control early muscle development.

Members of the MADS/MEF2 family of transcription factors, together with the MRFs, cooperatively control vertebrate myogenesis (Molkentin and Olson 1996; Yun and Wold 1996). The C. elegans MADS-box (MCM1, Agamous, Deficiens, and serum response factor) transcription factor unc-120 was first identified in a forward genetic screen for mutants with disrupted muscle function (Williams and Waterston 1994). In addition to postembryonic morphological and locomotory defects resulting from impaired muscle function, mutation of unc-120 was found to result in low penetrance embryonic arrest with a terminal phenotype paralyzed at the twofold stage (Pat), indicating a near complete lack of muscle function (Williams and Waterston 1994). The low penetrance Pat phenotype and post-embryonic defects are reminiscent of hlh-1 mutants (Chen et al. 1992, 1994), and subsequent molecular and cytological characterization of an unc-120 mutant revealed subtle and variable defects in differentiated muscle cells, in spite of grossly normal muscle specification (Caldicott and Waterston 1991). Given the critical role of the MADS-box-type transcription factor MEF2 in vertebrate and fly myogenesis (Bour et al. 1995; Lilly et al. 1995; Molkentin et al. 1995), its C. elegans ortholog, mef-2, was investigated as a candidate regulator of myogenesis, but a null mutation in mef-2 does not disrupt normal myogenesis, nor does it enhance the hlh-1 phenotype (Dichoso et al. 2000). Dichoso et al. (2000) tried RNA interference (RNAi) of unc-120 in a mef-2 mutant and did not find evidence for genetic redundancy between the two.

In addition to the MyoD and MADS/MEF2 families of proteins, HAND transcription factors have also been implicated in control of muscle development, although their role is largely restricted to cardiac muscle development (Cserjesi et al. 1995; Srivastava et al. 1995; Kolsch and Paululat 2002). The C. elegans genome contains a single HAND bHLH transcription factor, hnd-1. C. elegans does not have a heart, although the continuously pumping pharynx is considered a physiological analog, and the genetics of pharyngeal muscle development are consistent with such a parallel (Okkema et al. 1997). However, hnd-1 is not expressed in the pharynx, and loss of hnd-1 function does not affect pharyngeal muscle development (Mathies et al. 2003). hnd-1 is required for the development of other mesodermal cells; in particular, in hnd-1 mutants, the presence and position of somatic gonadal precursor cells is disrupted (Mathies et al. 2003). Leaving no stone unturned, Mathies et al. (2003) characterized the complete expression pattern of hnd-1 by reporter gene analysis, noting that in the early embryo, it is expressed exclusively in the myoblasts. Low-penetrance embryonic arrest was also noted for the hnd-1 mutant, which together with its expression pattern and the fact that it contains a bHLH domain led Mathies et al. (2003) to hypothesize that hnd-1 contributes to muscle specification by functioning redundantly with hlh-1. However, redundancy between the two was not convincingly detected, and they were conservatively reported not to have overlapping function (Mathies et al. 2003). Like the negative results of Dichoso et al. (2000) with mef-2 and unc-120, the reported lack of genetic redundancy between hlh-1 and hnd-1 supported the idea that control of muscle specification in C. elegans must somehow be different from vertebrates, apparently having diverged and come to rely on distinct, still unidentified factors functioning redundantly with hlh-1.

\section{Redundant specification of muscle by hlh-1, unc-120, and hnd-1}

In spite of its function not being essential to muscle specification in C. elegans, ectopic overexpression of HLH-1 in early embryonic blastomeres was recently shown by Fukushige and Krause (2005) to transform almost all cells into muscle independent of lineage, clearly demonstrating that like its vertebrate and fly counterparts, hlh-1 can direct myogenesis. This result is not unlike a variety of maternal effect mutations that transform embryonic blastomere identity, in turn affecting the number of muscle cells born in the embryo (Bowerman 1998). In particular, mutation of mex-3 produces dead embryos with an excess of muscle by derepressing PAL-1 function in the anterior of the early embryo, thus allowing it to transform the $\mathrm{AB}$ lineage into eight " $\mathrm{C}$ like" lineages (Draper et al. 1996; Hunter and Kenyon 1996). Consistent with transformation of distinct cell 
lineages into muscle by PAL-1 activity, hlh-1 expression is positively regulated by PAL-1 (Baugh et al. 2005a; Fukushige and Krause 2005).

Baugh et al. (2005a) identified hlh-1 in a microarray analysis of mRNA expression aimed at identifying PAL-1 transcriptional regulatory targets using mutant embryos with either no [pie-1 ${ }^{-/-}$; pal-1(RNAi)] or excess PAL-1 activity [mex-3-1-; skn-1(RNAi)], and in the same experiment unc-120 and hnd-1 were also identified. Using reporter gene analysis and RNAi of pal-1 and its repressor mex-3; hlh-1, unc-120, and hnd-1 were each shown to be expressed in early embryonic myoblasts under the control of PAL-1 (Baugh et al. 2005a). The overlapping temporal and spatial expression patterns, common upstream regulation, and similar mutant phenotypes of hlh-1 and unc-120 (Caldicott and Waterston 1991; Chen et al. 1992, 1994; Williams and Waterston 1994) led to the suggestion that hlh-1, unc-120, and hnd-1 function together to control muscle specification (Baugh et al. 2005a). In a subsequent study, synthetic lethality was systematically investigated for all pairs of validated PAL-1 targets in an effort to discover functions that are hidden from normal genetic screens by functional redundancy (Baugh et al. 2005b). Using different combinations of mutants and RNAi, a set of reciprocal, compensatory genetic interactions was identified among hlh-1, unc-120, and hnd-1, and the three genes were dubbed a "muscle module." Phenotypes resulting from single and double loss of function were quantified by scoring embryonic lethality, and a statistical model was used to evaluate epistasis. Although molecular markers of muscle development were not scored, carefully staged terminal embryos were found to exclusively have a Pat phenotype, indicating a lack of embryonic muscle function (Williams and Waterston 1994), which suggests that the observed synthetic lethality is due to a specific disruption of muscle development (Baugh et al. 2005b).

In this issue of Genes \& Development, Fukushige et al. (2006) present new data taking advantage of molecular markers and new mutations in hlh-1 and unc-120 to elucidate the genetic control of muscle specification in C. elegans. Like Baugh et al. (2005a), Fukushige et al. (2006) identified unc-120 and hnd-1 as regulatory targets of PAL-1 in a microarray mRNA expression analysis, except in this case using ectopic overexpression of PAL-1 from a heat-shock promoter as opposed to mutants that alter PAL-1 activity by disrupting its natural repressors. That these two critical PAL-1 targets were identified in both analyses reflects well on the results of each and underscores the value of such genome-wide approaches when executed effectively. Above all else, Fukushige et al. (2006) used genetic analysis to clearly demonstrate that hlh-1, unc-120, and hnd-1 function redundantly to specify body wall muscle during $C$. elegans embryogenesis, thus corroborating prior results and the suggestion that they comprise a "muscle module" (Baugh et al. 2005b). In contrast to previous work (Baugh et al. 2005b), Fukushige et al. (2006) demonstrate and characterize each of the three genetic interactions using double mutants as opposed to RNAi in a mutant background. Fur- thermore, expression of molecular markers of muscle differentiation was scored in addition to embryonic lethality. The results clearly demonstrate a nonadditive, synthetic phenotype specifically affecting muscle development in each of the double-mutant combinations. In addition, Fukushige et al. (2006) performed RNAi in the double-mutant backgrounds and scored, for the first time, the triple loss-of-function phenotype revealing a complete lack of muscle development. This result was confirmed by creating the triple loss of function in three different ways; that is, by performing RNAi of each of the three genes in each of the appropriate double-mutant backgrounds. Based on expression of molecular markers of differentiation, there is no specification of body wall muscle in any of the triple loss-of-function combinations, demonstrating for the first time a genetic loss of function resulting in complete disruption of C. elegans body wall muscle development.

In addition to loss-of-function analysis, Fukushige et al. (2006) investigate the developmental effects of ectopic overexpression of HLH-1, UNC-120, and HND-1. As previously described for HLH-1 (Fukushige and Krause 2005), a ubiquitously active heat-shock promoter was used to drive ectopic expression. Overexpression of each of the three factors alone can transform nearly all cells into muscle independent of lineage, indicating that each gene is a bona fide myogenic regulator. Fukushige et al. (2006) also explored epistasis of the three factors by overexpressing them in mutant backgrounds. Interestingly, they find that transformation into muscle by UNC-120 depends on hlh-1 function but that transformation by HND-1 does not depend on hlh-1. These results, together with additional microarray analysis of HLH-1 overexpression suggesting that unc-120 is a target of HLH-1, led Fukushige et al. (2006) to suggest that, like their vertebrate counterparts, hlh-1 and unc-120 activate each other's expression, rendering specification irreversible once under way. In contrast, they argue that hnd-1 only functions early in myogenesis to complement hlh-1 function probably by binding similar DNA sites via its bHLH domain. With ample evidence, both molecular and genetic, from multiple labs, it is now certain that $C$. elegans body wall muscle is specified by hlh-1, unc-120, and hnd-1. Thus, what was once a paradox in the genetic control of body wall muscle specification in C. elegans is now resolved.

\section{Conservation of myogenic regulators and robust regulatory relationships}

The overlapping function and reciprocal regulation among these three genes suggests that they form a "muscle module" with important implications for the developmental robustness and evolution of myogenesis. Although the single MyoD homolog hlh-1 is not essential to muscle specification, it does play a leading role together with two other conserved myogenic regulators, unc-120 and hnd-1. However, the fly orthologs of unc120 and hnd-1 do not play critical roles in body wall or skeletal myogenesis, but instead predominantly affect 
smooth and cardiac muscle development. This finding led Fukushige et al. (2006) to propose a single evolutionary origin for all contractile cell types, as opposed to multiple, independent origins and convergent evolution (see Fig. 1). Fukushige et al. (2006) argue that these regulators were present and functioning in combination to control development of the earliest muscle cells, and that gene duplications and other types of mutations followed, providing natural selection with the variation needed to evolve different types of muscle under diverse regulation. Consistent with this view, homologs of MyoD and MEF2 are expressed in the myogenic precursors of jellyfish, the most ancestral extant animal with muscle (Spring et al. 2002; Muller et al. 2003). Further support for this model may be obtained from solving the same paradox in D. melanogaster, where the single MyoD homolog, nautilus, is also not required for body wall muscle specification (Paterson et al. 1991). As is the case for its vertebrate counterpart, and probably also UNC-120, D. melanogaster Mef2 has recently been shown to transcriptionally activate nautilus, apparently playing a critical role early in myogenesis (Sandmann et al. 2006) and suggesting that it may function redundantly with nautilus, and possibly other factors, in early muscle development.

Perhaps what is most striking about the specification of C. elegans body wall muscle is the apparent conservation of overlapping function and reciprocal cross-regulation among multiple myogenic regulators. While the specific factors involved may vary in each case, a theme of myogenesis in every animal examined is its difficulty to disrupt, given redundancy of function and regulation. As first suggested by Weintraub (1993), what on the surface seems like a complex network, in fact, has the simple function of irreversibly committing cells to the myogenic pathway once specified. As demonstrated in bacteria, the specific organization of regulatory relationships in transcriptional circuits (topology) affects both the dynamics and robustness of circuit function (Alon 2006). We propose that muscle specification has continued to rely on such a set of redundant regulatory relationships as those exemplified in the muscle module of C. elegans because of the developmental robustness they confer. As indicated by years of genetic analysis that was unable to completely disrupt myogenesis, the muscle module is robust to the effects of mutation and probably also to environmental variation and molecular developmental noise. The beauty of the muscle module is that when function of one of its components is compromised, the module can compensate in real time. HLH-1 and UNC-120 are likely to autoregulate, but functional compensation can also result, either, as is likely the case for hlh-1 and hnd-1, by binding the same DNA sites and regulating the same target genes, or, as is likely the case for hlh-1 and unc-120, by both activating each other's expression and by cooperatively regulating common target genes (Molkentin et al. 1995). Thus the modular function of hlh-1, unc-120, and hnd-1 renders specification of body wall muscle in C. elegans remarkably robust, suggesting an explanation for the apparent conser- vation of overlapping function and reciprocal cross-regulation among myogenic regulators.

Integrated theoretical, experimental, and comparative analyses are necessary to characterize the dynamics and evolution of myogenic regulatory networks. A complete understanding of the molecular basis of cell fate specification requires an understanding of the functional dynamics of gene regulation during specification. Models of myogenic regulatory networks can make quantitative predictions about the dynamics of gene expression and the consequences of network disruption; such predictions can then be tested by time-series analysis of gene expression and the models recursively optimized. Such combination of theory and experiment promises to put developmental robustness into real, experimental terms and in the process to foster a deeper understanding of the molecular basis of cell fate specification. The developmental robustness of myogenesis may, in fact, be what endowed the earliest myogenic regulatory networks with the evolvability necessary to give rise to multiple, distinct muscle types and a diversity of upstream activators (Kirschner and Gerhart 1998), but evidence for or against such a model will have to await more extensive comparative analyses between different types of muscle and different species.

\section{Acknowledgments}

We thank Paul Sternberg, Itai Yanai, and Steven Kuntz for help in revision. L.R.B. is supported by the American Cancer Society, and C.P.H. is funded by NIH GM064429.

\section{References}

Alon, U. 2006. An introduction to systems biology: Design principles of biological circuits. Chapman \& Hall, Boca Raton, FL.

Baugh, L.R., Hill, A.A., Claggett, J.M., Hill-Harfe, K., Wen, J.C., Slonim, D.K., Brown, E.L., and Hunter, C.P. 2005a. The homeodomain protein PAL-1 specifies a lineage-specific regulatory network in the C. elegans embryo. Development 132: 1843-1854.

Baugh, L.R., Wen, J.C., Hill, A.A., Slonim, D.K., Brown, E.L., and Hunter, C.P. 2005b. Synthetic lethal analysis of Caenorhabditis elegans posterior embryonic patterning genes identifies conserved genetic interactions. Genome Biol. 6: R45.

Bour, B.A., O'Brien, M.A., Lockwood, W.L., Goldstein, E.S., Bodmer, R., Taghert, P.H., Abmayr, S.M., and Nguyen, H.T. 1995. Drosophila MEF2, a transcription factor that is essential for myogenesis. Genes \& Dev. 9: 730-741.

Bowerman, B. 1998. Maternal control of pattern formation in early Caenorhabditis elegans embryos. Curr. Top. Dev. Biol. 39: 73-117.

Braun, T., Rudnicki, M.A., Arnold, H.H., and Jaenisch, R. 1992. Targeted inactivation of the muscle regulatory gene Myf-5 results in abnormal rib development and perinatal death. Cell 71: 369-382.

Caldicott, I.M. and Waterston, R.H. 1991. unc-120: Another gene with effects on muscle. Worm Breed. Gaz. 11: 98.

Chen, L., Krause, M., Draper, B., Weintraub, H., and Fire, A. 1992. Body-wall muscle formation in Caenorhabditis elegans embryos that lack the MyoD homolog hlh-1. Science 
256: 240-243.

Chen, L., Krause, M., Sepanski, M., and Fire, A. 1994. The Caenorhabditis elegans MYOD homologue HLH-1 is essential for proper muscle function and complete morphogenesis. Development 120: 1631-1641.

Cserjesi, P., Brown, D., Lyons, G.E., and Olson, E.N. 1995. Expression of the novel basic helix-loop-helix gene eHAND in neural crest derivatives and extraembryonic membranes during mouse development. Dev. Biol. 170: 664-678.

Dichoso, D., Brodigan, T., Chwoe, K.Y., Lee, J.S., Llacer, R., Park, M., Corsi, A.K., Kostas, S.A., Fire, A., Ahnn, J., et al. 2000. The MADS-Box factor CeMEF2 is not essential for Caenorhabditis elegans myogenesis and development. Dev. Biol. 223: 431-440.

Draper, B.W., Mello, C.C., Bowerman, B., Hardin, J., and Priess, J.R. 1996. MEX-3 is a KH domain protein that regulates blastomere identity in early C. elegans embryos. Cell 87: 205216.

Fukushige, T. and Krause, M. 2005. The myogenic potency of HLH-1 reveals wide-spread developmental plasticity in early C. elegans embryos. Development 132: 1795-1805.

Fukushige, T., Brodigan, T.M., Schriefer, L.A., Waterston, R.H., and Krause, M. 2006. Defining the transcriptional redundancy of early bodywall muscle development in C. elegans: Evidence for a unified theory of animal muscle development. Genes \& Dev. (this issue).

Hunter, C.P. and Kenyon, C. 1996. Spatial and temporal controls target pal-1 blastomere-specification activity to a single blastomere lineage in C. elegans embryos. Cell 87: 217226.

Kablar, B., Krastel, K., Ying, C., Asakura, A., Tapscott, S.J., and Rudnicki, M.A. 1997. MyoD and Myf-5 differentially regulate the development of limb versus trunk skeletal muscle. Development 124: 4729-4738.

Kassar-Duchossoy, L., Gayraud-Morel, B., Gomes, D., Rocancourt, D., Buckingham, M., Shinin, V., and Tajbakhsh, S. 2004. Mrf4 determines skeletal muscle identity in Myf5:Myod double-mutant mice. Nature 431: 466-471.

Kirschner, M. and Gerhart, J. 1998. Evolvability. Proc. Nat1. Acad. Sci. 95: 8420-8427.

Kolsch, V. and Paululat, A. 2002. The highly conserved cardiogenic bHLH factor Hand is specifically expressed in circular visceral muscle progenitor cells and in all cell types of the dorsal vessel during Drosophila embryogenesis. Dev. Genes Evol. 212: 473-485.

Krause, M., Fire, A., Harrison, S.W., Priess, J., and Weintraub, H. 1990. CeMyoD accumulation defines the body wall muscle cell fate during C. elegans embryogenesis. Cell 63: 907-919.

Lilly, B., Zhao, B., Ranganayakulu, G., Paterson, B.M., Schulz, R.A., and Olson, E.N. 1995. Requirement of MADS domain transcription factor D-MEF2 for muscle formation in Drosophila. Science 267: 688-693.

Mak, K.L., To, R.Q., Kong, Y., and Konieczny, S.F. 1992. The MRF4 activation domain is required to induce muscle-specific gene expression. Mol. Cell. Biol. 12: 4334-4346.

Mathies, L.D., Henderson, S.T., and Kimble, J. 2003. The C. elegans Hand gene controls embryogenesis and early gonadogenesis. Development 130: 2881-2892.

Molkentin, J.D. and Olson, E.N. 1996. Combinatorial control of muscle development by basic helix-loop-helix and MADSbox transcription factors. Proc. Natl. Acad. Sci. 93: 93669373.

Molkentin, J.D., Black, B.L., Martin, J.F., and Olson, E.N. 1995. Cooperative activation of muscle gene expression by MEF2 and myogenic bHLH proteins. Cell 83: 1125-1136.

Muller, P., Seipel, K., Yanze, N., Reber-Muller, S., Streitwolf-
Engel, R., Stierwald, M., Spring, J., and Schmid, V. 2003 Evolutionary aspects of developmentally regulated helixloop-helix transcription factors in striated muscle of jellyfish. Dev. Biol. 255: 216-229.

Okkema, P.G., Ha, E., Haun, C., Chen, W., and Fire, A. 1997. The Caenorhabditis elegans NK-2 homeobox gene ceh-22 activates pharyngeal muscle gene expression in combination with pha- 1 and is required for normal pharyngeal development. Development 124: 3965-3973.

Ordahl, C.P. and Williams, B.A. 1998. Knowing chops from chuck: Roasting myoD redundancy. Bioessays 20: 357-362.

Paterson, B.M., Walldorf, U., Eldridge, J., Dubendorfer, A. Frasch, M., and Gehring, W.J. 1991. The Drosophila homologue of vertebrate myogenic-determination genes encodes a transiently expressed nuclear protein marking primary myogenic cells. Proc. Natl. Acad. Sci. 88: 3782-3786.

Rudnicki, M.A., Braun, T., Hinuma, S., and Jaenisch, R. 1992. Inactivation of MyoD in mice leads to up-regulation of the myogenic HLH gene Myf-5 and results in apparently normal muscle development. Cell 71: 383-390.

Rudnicki, M.A., Schnegelsberg, P.N., Stead, R.H., Braun, T., Arnold, H.H., and Jaenisch, R. 1993. MyoD or Myf-5 is required for the formation of skeletal muscle. Cell 75: 1351-1359.

Sandmann, T., Jensen, L.J., Jakobsen, J.S., Karzynski, M.M., Eichenlaub, M.P., Bork, P., and Furlong, E.E. 2006. A temporal map of transcription factor activity: mef2 directly regulates target genes at all stages of muscle development. Dev. Cell 10: $797-807$.

Spring, J., Yanze, N., Josch, C., Middel, A.M., Winninger, B., and Schmid, V. 2002. Conservation of Brachyury, Mef2, and Snail in the myogenic lineage of jellyfish: A connection to the mesoderm of bilateria. Dev. Biol. 244: 372-384.

Srivastava, D., Cserjesi, P., and Olson, E.N. 1995. A subclass of bHLH proteins required for cardiac morphogenesis. Science 270: 1995-1999.

Weintraub, H. 1993. The MyoD family and myogenesis: Redundancy, networks, and thresholds. Cell 75: 1241-1244.

Weintraub, H., Tapscott, S.J., Davis, R.L., Thayer, M.J., Adam, M.A., Lassar, A.B., and Miller, A.D. 1989. Activation of muscle-specific genes in pigment, nerve, fat, liver, and fibroblast cell lines by forced expression of MyoD. Proc. Natl. Acad. Sci. 86: 5434-5438.

Williams, B.D. and Waterston, R.H. 1994. Genes critical for muscle development and function in Caenorhabditis elegans identified through lethal mutations. J. Cell Biol. 124: 475-490.

Yun, K. and Wold, B. 1996. Skeletal muscle determination and differentiation: Story of a core regulatory network and its context. Curr. Opin. Cell Biol. 8: 877-889. 


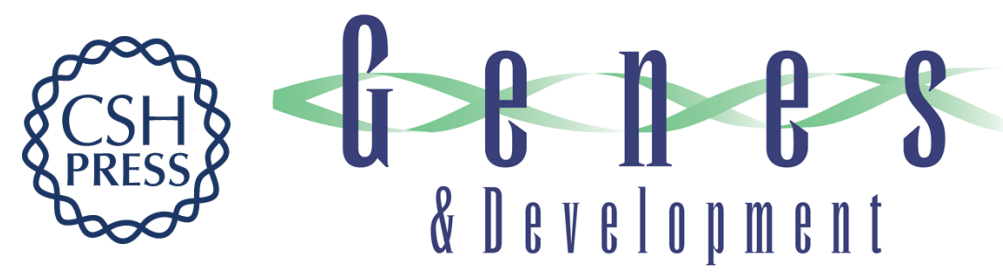

\section{MyoD, modularity, and myogenesis: conservation of regulators and redundancy in C. elegans}

L. Ryan Baugh and Craig P. Hunter

Genes Dev. 2006, 20:

Access the most recent version at doi:10.1101/gad.1507606

Related Content Defining the transcriptional redundancy of early bodywall muscle development in C. elegans: evidence for a unified theory of animal muscle development

Tetsunari Fukushige, Thomas M. Brodigan, Lawrence A. Schriefer, et al.

Genes Dev. December , 2006 20: 3395-3406

References This article cites 36 articles, 16 of which can be accessed free at:

http://genesdev.cshlp.org/content/20/24/3342.full.html\#ref-list-1

Articles cited in:

http://genesdev.cshlp.org/content/20/24/3342.full.html\#related-urls

\section{License}

Email Alerting

Service

Receive free email alerts when new articles cite this article - sign up in the box at the top right corner of the article or click here.

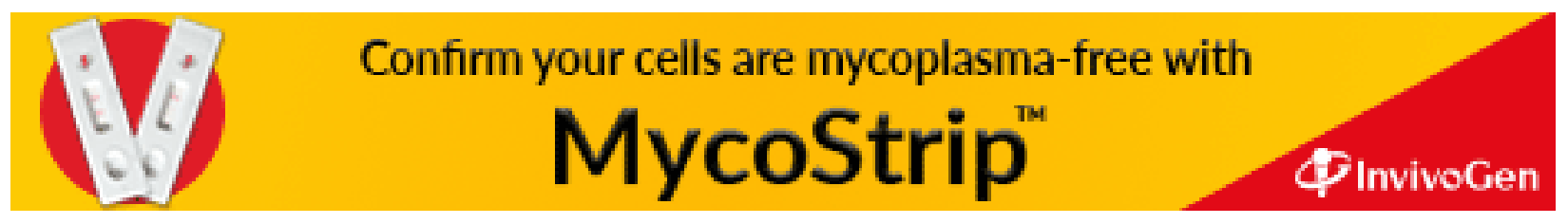

